\title{
Properties and removal of singular couplings at conical intersections
}

\author{
L. F. Errea, A. Macías, * L. Méndez, I. Rabadán, A. Riera, A. Rojas, and P. Sanz \\ Departamento de Química, C-IX, Universidad Autónoma de Madrid, Canto Blanco, 28049 Madrid, Spain
}

(Received 18 December 2000; published 10 May 2001)

\begin{abstract}
We present an analysis of the characteristics of nonadiabatic couplings due to the existence of conical intersections between potential energy surfaces of triatomic systems in collinear configurations. We discuss the relative merits and performance of four techniques that we tested to remove the singularities, and illustrate our findings for a conical point of two molecular surfaces involved in $\mathrm{N}^{5+}+\mathrm{H}_{2}$ collisions.
\end{abstract}

DOI: 10.1103/PhysRevA.63.062713

PACS number(s): 34.50.-s, 34.70.+e, 34.10.+x

\section{INTRODUCTION}

As is well known, transitions at conical intersections (CIs) of potential energy surfaces provide a standard mechanism for nonradiative processes at very low energies. They are also ubiquitous in the treatment of ion-(atom-)diatomic reactions at higher energies involving near-collinear and nearperpendicular trajectories, as was seen in our study of electron capture from $\mathrm{H}_{2}$ targets by ion impact (e.g., Refs. [1-3]), which is an important topic in fusion research [4]. In addition, there are many advantages in starting a dynamical study with collinear trajectories, for which wave functions have an additional symmetry (see below) and therefore the \pm sign ambiguities of the nonadiabatic couplings at avoided crossings are resolved with less difficulty. Hence, the problem is certainly not only of academic interest.

The first question that comes to mind in considering CIs is whether geometric phases [5,7-9] are required to treat the dynamics. In Ref. [10], we showed how these phases can be obviated for open trajectories, and that the practical difficulty is the singular character of dynamical couplings at the CIs. This singular character precludes a direct use of the adiabatic wave functions in close-coupling treatments; the implications of infinitely large couplings, even for single-state dynamics, have been stressed by Baer and co-workers (see, e.g., Ref. [6]). Rotational couplings are particularly difficult: they exhibit a pole at the CI that gives rise to a logarithmic singularity of the exact transition probability amplitude for a head-on trajectory; moreover, the effect of the CI can extend to regions far away from it, where it is difficult to implement even an approximate, ad hoc elimination of the corresponding coupling.

A preliminary analysis of the singularity problem was given in Ref. [10], and complemented previous work of $[7,11]$ for near-equilateral-triangle nuclear configurations, and of Ref. [12] by considering also the rotational couplings. The theoretical framework of Ref. [10] was our implementation [13] of the sudden approximation model for ion impact on diatomic molecules, which has been succcessfully applied to a sizable number of reactions $[19,1,14-16,2,3,17]$. In this model, the vibrorotational structure of the target is assumed

\footnotetext{
*Also at Instituto de Estructura de la Materia, CSIC, Serrano 113 bis, 28006 Madrid, Spain.
}

to be "frozen" while the electron capture process takes place. To describe this process, an extension of the standard treatment of ion-atom collisions [18] is employed: the ion follows a trajectory $\boldsymbol{R}(t)$ while the target internuclear vector $\boldsymbol{\rho}$ stays fixed; and the electronic structure is described using a close-coupling expansion in terms of the eigenfunctions of the triatomica molecule. These eigenfunctions are defined for fixed positions of the nuclei in the $(\boldsymbol{\rho}, \boldsymbol{R})$ plane. An increased symmetry is obtained in the collinear configuration, such that $\boldsymbol{R} \| \boldsymbol{\rho}$, and we have $\Sigma, \Pi, \Delta$, etc., states. Then, any of the usual $\Sigma-\Pi, \Sigma-\Delta$, etc., energy crossings becomes a CI of the corresponding energy surface for more general geometries, where radial and rotational components of the nonadiabatic coupling operator $\boldsymbol{\nabla}_{R}$ are singular [10].

An implication of these findings for the best known method to treat ion-molecule dynamics at very low impact velocities, which is the infinite-order sudden approximation [12], may be mentioned. In this method, rotational couplings are neglected, so that the troublesome rotational singularities are absent; nevertheless, those of the radial couplings remain. At higher velocities such as those considered here, rotational couplings are often very important, and both singularities must be removed.

In the next section we present a more detailed account of the theory than in our previous paper [10], showing the physical origin of the singularities. As a side result, we find that the matrix elements of the nonadiabatic vibrational coupling term $\boldsymbol{\nabla}_{\rho}$ also become infinite at CIs. This contradicts the basic tenets of our sudden approximation approach (which neglects these couplings), and stresses again the need for removing the singularities before using this approach. In our previous work, a parametrization of the adiabatic data was employed to cancel the singularities, and illustrated for the case of $\mathrm{Li}^{+}+\mathrm{H}_{2}$ collisions; the procedure was also recently applied to charge transfer in the $\mathrm{N}^{5+}+\mathrm{H}_{2}$ reaction [19], for which experimental data have recently been reported [20]. However, this method is difficult to implement because it is extremely sensitive to the values of the parameters, and in particular it is impracticable when CIs are not completely isolated. Because of this, we have considered several alternative approaches. In Sec. III, we report our findings on their applicability; for the sake of clarity, our procedure is illustrated for an isolated $\mathrm{CI}$ of the $\mathrm{NH}_{2}{ }^{5+}$ quasimolecule, although the more general situation is kept in mind. Our conclusions are drawn in Sec. IV.

Atomic units are employed throughout. 


\section{THEORY}

\section{A. Couplings in the laboratory-fixed and body-fixed frames of reference}

We shall follow here the theoretical framework given in Ref. [10]: we have, in a laboratory-fixed reference frame, the variables $\boldsymbol{R}$, defining the position of the projectile with respect to the diatomic target, $\boldsymbol{\rho}$, the internuclear vector of the target, and $\boldsymbol{r}_{i}$, the position of the electrons. Taking the $Z$ axis along $\boldsymbol{\rho}$, and with $R, \alpha$ the polar coordinates of $\boldsymbol{R}$ in the $X Z$ plane, the Cartesian coordinates of those vectors are

$$
\boldsymbol{r}_{i}=\left(x_{i}, y_{i}, z_{i}\right), \quad \boldsymbol{R}=(R \sin \alpha, 0, R \cos \alpha), \quad \boldsymbol{\rho}=(0,0, \rho) .
$$

We further define a rotation operator $\mathfrak{R}(\omega)$ of an angle $\omega$ about the $Y$ axis, and a rotated body-fixed frame in which the new $\bar{Z}$ axis lies along $\boldsymbol{R}$, and the new position vectors are $\overline{\boldsymbol{R}}=\mathfrak{R}(-\alpha) \boldsymbol{R}, \overline{\boldsymbol{\rho}}=\mathfrak{R}(-\alpha) \boldsymbol{\rho}, \overline{\boldsymbol{r}}_{i}=\mathfrak{R}(-\alpha) \boldsymbol{r}_{i}:$

$$
\begin{aligned}
\overline{\boldsymbol{r}}_{i} & =\left(x_{i} \cos \alpha-z_{i} \sin \alpha, y_{i}, x_{i} \sin \alpha+z_{i} \cos \alpha\right), \\
\overline{\boldsymbol{R}} & =(0,0, R), \quad \overline{\boldsymbol{\rho}}=\left(-\rho \sin \alpha=\rho \sin \rho_{\alpha}, 0, \rho \cos \alpha\right. \\
& \left.=\rho \cos \rho_{\alpha}\right) .
\end{aligned}
$$

Since the electronic wave functions depend only on relative coordinates, we have, for any angle $\omega$,

$$
\psi(\boldsymbol{r} ; \boldsymbol{R} ; \boldsymbol{\rho})=\psi(\mathfrak{R}(\omega) \boldsymbol{r} ; \mathfrak{R}(\omega) \boldsymbol{R} ; \mathfrak{R}(\omega) \boldsymbol{\rho})=\psi(\overline{\boldsymbol{r}} ; \overline{\boldsymbol{R}} ; \overline{\boldsymbol{\rho}})
$$

We now consider the nuclear gradient $\nabla_{R}$ coupling between two such wave functions. Using a compact notation, we write the partial derivative with respect to $R$ as $\partial_{R}^{\{\boldsymbol{r}, \alpha, \boldsymbol{\rho}\}}$ keeping constant the set of electronic coordinates $\boldsymbol{r}$, the polar angle $\alpha$, and the nuclear vector $\boldsymbol{\rho}$. To be specific, we take a trajectory of the projectile $\boldsymbol{R}(t)$ in the $X Z$ collision plane, so that we have to consider two components of the coupling operator:

$$
\nabla_{R}=\hat{R} \partial_{R}^{\{\boldsymbol{r}, \alpha, \boldsymbol{\rho}\}}+\hat{\alpha} R^{-1} \partial_{\alpha}^{\{\boldsymbol{r}, R, \boldsymbol{\rho}\}}
$$

We now transform to the body-fixed frame. Since the rotation leaves $R$ invariant, from the property (3) of the wave function, we have

$$
\partial_{R}^{\{\boldsymbol{r}, \alpha, \boldsymbol{\rho}\}} \psi=\partial_{R}^{\{\overline{\boldsymbol{r}}, \overline{\boldsymbol{\rho}}\}} \psi
$$

The tranformation for the rotational coupling is slightly more complicated. To order $\delta \alpha$, we have, using Eqs. (2) and (3),

$$
\begin{aligned}
\delta \alpha \partial_{\alpha}^{\{\boldsymbol{r}, R, \boldsymbol{\rho}\}} \psi & =\psi(\boldsymbol{r} ; \Re(-\delta \alpha) \boldsymbol{R} ; \boldsymbol{\rho})-\psi(\boldsymbol{r} ; \boldsymbol{R} ; \boldsymbol{\rho}) \\
& =\psi(\Re(-\alpha-\delta \alpha) \boldsymbol{r} ; \overline{\boldsymbol{R}} ; \Re(-\delta \alpha) \overline{\boldsymbol{\rho}})-\psi(\Re(-\alpha) \boldsymbol{r} ; \overline{\boldsymbol{R}} ; \overline{\boldsymbol{\rho}}) \\
& =\psi(\Re(-\alpha-\delta \alpha) \boldsymbol{r} ; \overline{\boldsymbol{R}} ; \Re(-\delta \alpha) \overline{\boldsymbol{\rho}})-\psi(\Re(-\alpha-\delta \alpha) \boldsymbol{r} ; \overline{\boldsymbol{R}} ; \overline{\boldsymbol{\rho}})+\psi(\Re(-\alpha-\delta \alpha) \boldsymbol{r} ; \overline{\boldsymbol{R}} ; \overline{\boldsymbol{\rho}})-\psi(\Re(-\alpha) \boldsymbol{r} ; \overline{\boldsymbol{R}} ; \overline{\boldsymbol{\rho}}) \\
& =-\delta \alpha \partial_{\rho_{\alpha}\{\overline{\boldsymbol{r}}, \overline{\boldsymbol{R}}, \rho\}}^{\}} \psi(\overline{\boldsymbol{r}} ; \overline{\boldsymbol{R}} ; \overline{\boldsymbol{\rho}})-\delta \alpha \sum_{i} \partial_{\bar{r}_{i}}^{\{\overline{\boldsymbol{R}}, \overline{\boldsymbol{\rho}}\}} \psi(\overline{\boldsymbol{r}} ; \overline{\boldsymbol{R}} ; \overline{\boldsymbol{\rho}}) \cdot \partial_{\alpha}^{\{\boldsymbol{r}, \overline{\boldsymbol{R}}, \overline{\boldsymbol{\rho}}\}} \overline{\boldsymbol{r}}_{i},
\end{aligned}
$$

and from Eq. (2)

$$
\begin{aligned}
\partial_{\alpha}^{\{\boldsymbol{r}, R, \boldsymbol{\rho}\}} \psi= & -\partial_{\rho_{\alpha}}^{\{\overline{\boldsymbol{r}}, \overline{\boldsymbol{R}}, \rho\}} \psi-\sum_{i}\left(-\bar{z}_{i} \partial_{\bar{x}_{i}}^{\left\{\bar{y}_{i}, \bar{z}_{i}, \overline{\boldsymbol{r}}_{j}, \overline{\boldsymbol{R}}, \overline{\boldsymbol{\rho}}\right\}}\right. \\
& \left.+\bar{x}_{i} \partial_{\bar{z}_{i}}^{\left\{\bar{x}_{i}, \bar{y}_{i}, \overline{\boldsymbol{r}}_{j}, \overline{\boldsymbol{R}}, \overline{\boldsymbol{\rho}}\right\}}\right) \psi \\
= & -\partial_{\rho_{\alpha}}^{\{\overline{\boldsymbol{r}, \boldsymbol{R}}, \rho\}} \psi(\overline{\boldsymbol{r}} ; \overline{\boldsymbol{R}} ; \overline{\boldsymbol{\rho}})+i \quad L_{\bar{y}} \psi(\overline{\boldsymbol{r}} ; \overline{\boldsymbol{R}} ; \overline{\boldsymbol{\rho}})
\end{aligned}
$$

with $L_{y}$ the component of the total electronic angular momentum along the $Y=\bar{Y}$ axis. Using Eqs. (4), (5), and (7), we obtain

$$
\left.\nabla_{R} \psi=\left[\hat{R} \partial_{R}^{\{\bar{r}, \overline{\boldsymbol{\rho}}}\right\}+\hat{\alpha} R^{-1}\left(i \quad L_{y}^{-}-\partial_{\rho_{\alpha}}^{\{\overline{\boldsymbol{r}}, \overline{\boldsymbol{R}}, \rho\}}\right)\right] \psi
$$

This expression, which was not explicitly derived in Refs. $[13,10]$, shows that the mechanisms of ion-molecule collisions differ from those of ion-atom dynamics by transitions due to the couplings through the $\partial_{\rho_{\alpha}}^{\{\overline{\boldsymbol{r}}, \overline{\boldsymbol{R}}, \rho\}}$ operator. The other two operators $\partial_{R}^{\{\overline{\boldsymbol{r}}, \overline{\boldsymbol{\rho}}\}}$ and $i L_{y}^{-}$yield the usual radial and rotational couplings, respectively. They arise from the changes in the wave functions with $R$ (radial coupling) and $\alpha$ (rotational coupling) for fixed electronic and $\boldsymbol{\rho}$ positions. The $\partial_{\rho_{\alpha}}^{\{\overline{\boldsymbol{r}}, \overline{\boldsymbol{R}}, \rho\}}$ operator arises from the rotation of the diatomic molecule by an angle $\rho_{\alpha}=-\alpha$ for fixed projectile and electronic positions, and is at the root of the singular character of the rotational couplings at CIs. This will be explicitly shown below, but is already apparent from Eq. (7), together with the fact that the interactions through the $i L_{\bar{y}}$ operator (which involves derivatives with respect to the electronic coordinates) cannot be singular, even when the corresponding wave functions change their character abruptly as functions of $\boldsymbol{R}$ or $\boldsymbol{\rho}$. Hence, any singularity must be attributed to the first term in Eq. (7).

\section{B. Singular couplings at conical intersections}

In Ref. [10] we considered a CI of two energy surfaces $E_{1}(\boldsymbol{R} ; \boldsymbol{\rho})$ and $E_{2}(\boldsymbol{R} ; \boldsymbol{\rho})$ at a point $\boldsymbol{R}=\boldsymbol{R}_{0}$ of the $Z$ axis, and 
studied the analytical behavior of the dynamical couplings near this CI. For this, we employed the standard procedure of a Smith transformation [21]

$$
\begin{aligned}
& \psi_{1}=(\cos \theta) \varphi_{1}+(\sin \theta) \varphi_{2}, \\
& \psi_{2}=-(\sin \theta) \varphi_{1}+(\cos \theta) \varphi_{2}
\end{aligned}
$$

in terms of two functions $\varphi_{1}, \varphi_{2}$ that are smoothly varyingwhich means that their nonadiabatic couplings do not present singularities, not that they vanish: hence, the new functions are not necessarily diabatic. Use of a bilinear expansion in $R-R_{0}$ and $R|\alpha|$ for the Hamiltonian matrix elements in this basis [10] yields the limit expression for the transformation angle near the $\mathrm{CI}$ :

$$
\theta=\frac{\pi}{4}+\frac{1}{2} \tan ^{-1} \frac{a\left(R-R_{0}\right)+b R|\alpha|}{2 c R|\alpha|}
$$

Using this, it follows that, as $\boldsymbol{R} \rightarrow \boldsymbol{R}_{0}$ (so that $R \rightarrow R_{0}$ and $\alpha \rightarrow 0$ ) the limit behavior of the radial and rotational couplings follows from Eq. (4) and the expressions

$$
\begin{aligned}
\left\langle\psi_{1}\left|\partial_{R}^{\left\{r, \alpha, \boldsymbol{\rho}_{\}}\right.}\right| \psi_{2}\right\rangle= & \partial_{R}^{\left\{\alpha, \boldsymbol{\rho}_{\}}\right.} \theta+(\text { regular terms }) \\
= & -\frac{a c R_{0}|\alpha|}{4 c^{2} R^{2} \alpha^{2}+\left[a\left(R-R_{0}\right)+b R|\alpha|\right]^{2}} \\
& +(\text { regular terms })
\end{aligned}
$$

and

$$
\begin{aligned}
\left\langle\psi_{1}\left|\partial_{\alpha}^{\{r, R, \boldsymbol{\rho}\}}\right| \psi_{2}\right\rangle= & \partial_{\alpha}^{\{R, \boldsymbol{\rho}\}} \theta+(\text { regular terms }) \\
= & \operatorname{sgn}(\alpha) \frac{a c R\left(R-R_{0}\right)}{4 c^{2} R^{2} \alpha^{2}+\left[a\left(R-R_{0}\right)+b R|\alpha|\right]^{2}} \\
& +(\text { regular terms }),
\end{aligned}
$$

where the parameters $R_{0}, a, b$, and $c$ can be obtained by fitting the energy difference to the well-known double cone form in the neighborhood of the CI:

$$
E_{2}-E_{1}=\sqrt{\left[a\left(R-R_{0}\right)+b R|\alpha|\right]^{2}+4 c^{2} R^{2} \alpha^{2}} .
$$

We can now change to the body-fixed frame, by using Eqs. (5) and (7), to obtain the limit expressions near the CI:

$$
\begin{aligned}
\left\langle\psi_{1}\left|\partial_{R}^{\{\bar{r}, \overline{\boldsymbol{\rho}}\}}\right| \psi_{2}\right\rangle= & \left\langle\psi_{1}\left|\partial_{R}^{\left\{\boldsymbol{r}, \alpha, \boldsymbol{\rho}_{\}}\right.}\right| \psi_{2}\right\rangle=\partial_{R}^{\{\overline{\boldsymbol{\rho}}\}} \theta+(\text { regular terms }) \\
= & -\frac{a c R_{0}|\alpha|}{4 c^{2} R^{2} \alpha^{2}+\left[a\left(R-R_{0}\right)+b R|\alpha|\right]^{2}} \\
& +(\text { regular terms })
\end{aligned}
$$

with $\partial_{R}^{\{\overline{\boldsymbol{\rho}}\}} \theta=\partial_{R}^{\{\alpha, \boldsymbol{\rho}\}} \theta$, and

$$
\begin{aligned}
\left\langle\psi_{1}\left|\partial_{\rho_{\alpha}}^{\{\bar{r}, \overline{\boldsymbol{R}}, \rho\}}\right| \psi_{2}\right\rangle= & -\left\langle\psi_{1}\left|\partial_{\alpha}^{\{r, R, \boldsymbol{\rho}\}}\right| \psi_{2}\right\rangle+\left\langle\psi_{1}\left|i L_{y}^{-}\right| \psi_{2}\right\rangle \\
= & \partial_{\rho_{\alpha}}^{\{\overline{\boldsymbol{R}}, \rho\}} \theta+(\text { regular terms }) \\
= & \operatorname{sgn}(\alpha) \frac{-a c R\left(R-R_{0}\right)}{4 c^{2} R^{2} \alpha^{2}+\left[a\left(R-R_{0}\right)+b R|\alpha|\right]^{2}} \\
& +(\text { regular terms }),
\end{aligned}
$$

where we have taken into account that, since $\left\langle\psi_{1}\left|i L_{y}^{-}\right| \psi_{2}\right\rangle$ is regular, the singular parts of $\left\langle\psi_{1}\left|\partial_{\rho_{\alpha}}^{\langle\overline{\boldsymbol{r}}, \overline{\boldsymbol{R}}, \rho\}}\right| \psi_{2}\right\rangle$ and $-\left\langle\psi_{1}\left|\partial_{\alpha}^{\{\boldsymbol{r}, R, \boldsymbol{\rho}\}}\right| \psi_{2}\right\rangle$ must be the same, and $\partial_{\boldsymbol{\rho}_{\alpha}}^{\left\{\overline{\boldsymbol{R}}, \rho^{\alpha}\right\}} \theta=-\partial_{\alpha}^{\{R, \boldsymbol{\rho}\}} \theta$.

When $\alpha \rightarrow 0$, and as functions of $R$, the radial coupling (11) tends to a $\delta$ function, while the rotational coupling (12) gives rise to both a $\delta$ function and a pole $c R_{0} a^{-1}(R$ $\left.-R_{0}\right)^{-1}$. The two singularities are connected, as shown in Ref. [10] by examining the behavior of the nuclear gradient in terms of the variable $\left\{2 c a^{-1} R|\alpha|, a(R-\alpha)+b R|\alpha|\right\}$, and this is why elimination of one of the singularities entails supression of the other one, as was confirmed by our calculations $[10,19]$. As mentioned in the Introduction, the lefthand side (LHS) of Eq. (16) is the angular component of the matrix element $\left\langle\psi_{1}\left|\nabla_{\rho}\right| \psi_{2}\right\rangle$, which is therefore singular at the CI; moreover, the singularity of the radial component was shown in Ref. [12]. Hence, the coupling is far from being negligible as assumed in Ref. [13].

It is useful to introduce, at this stage, a simplification of these analytical expressions, obtained by expanding them about the position $R_{\alpha}$ of the peak of the radial coupling (11). In the laboratory-fixed frame, this yields the limit expression

$$
\begin{aligned}
\left\langle\psi_{1}\left|\partial_{R}^{\left\{\boldsymbol{r}, \alpha, \boldsymbol{\rho}_{\}}\right\}}\right| \psi_{2}\right\rangle & =\partial_{R}^{\left\{\alpha, \boldsymbol{\rho}_{\}}\right\}} \theta^{\prime}+(\text { regular terms }) \\
& =-\frac{\delta_{\alpha}|\alpha| / 2}{\left(R-R_{\alpha}\right)^{2}+\delta_{\alpha}^{2} \alpha^{2}}+(\text { regular terms })
\end{aligned}
$$

and

$$
\begin{aligned}
\left\langle\psi_{1}\left|\partial_{\alpha}^{\{r, R, \boldsymbol{\rho}\}}\right| \psi_{2}\right\rangle & =\partial_{\alpha}^{\{R, \boldsymbol{\rho}\}} \theta^{\prime}+(\text { regular terms }) \\
& =\frac{\delta_{\alpha}\left(R-R_{\alpha}\right) / 2}{\left(R-R_{\alpha}\right)^{2}+\delta_{\alpha}^{2} \alpha^{2}}+(\text { regular terms }) .
\end{aligned}
$$

The Landau-Zener type equation (17) (see also Ref. [12]) for the radial coupling, and Eq. (18) for the rotational term, contain only two parameters, $R_{\alpha}$ and $\delta_{\alpha}$ such that $\lim _{\alpha \rightarrow 0} R_{\alpha}$ $=R_{0}$ and $\lim _{\alpha \rightarrow 0} \delta_{\alpha}=\delta_{0}=2 c R_{0} / a$. For small $\alpha$, we have $R_{\alpha} \approx R_{0}-b R_{0} \alpha / a$ and $\delta_{\alpha} \approx \delta_{0}$.

Equations (17) and (18) yield a simpler behavior for the transformation angle, which we have called $\theta^{\prime}$ to avoid confusion with Eq. (10):

$$
\theta^{\prime}=\frac{\pi}{4}+\frac{1}{2} \tan ^{-1} \frac{R-R_{\alpha}}{\delta_{\alpha}|\alpha|},
$$


where the single branch $(-\pi / 2, \pi / 2)$ form of the $\tan ^{-1}$ function is implied, and which can be employed to explain the physical origin of the singularities in a simple way.

First, the behavior at the CI $\boldsymbol{R}=\boldsymbol{R}_{0}$ is easily obtained. The $\delta$ function in the radial coupling arises from the fact that, along the $Z$ axis, $\psi_{1}$ and $\psi_{2}$ have the same character as $\varphi_{1}$ and $\varphi_{2}$ for $R<R_{0}$ and the same as $\varphi_{2}$ and $-\varphi_{1}$ for $R>R_{0}$. There is a discontinuous change of character [22] at the CI and accordingly we have the asymptotic limit there:

$$
\partial_{R}^{\{\overline{\boldsymbol{\rho}}\}} \theta^{\prime}=\partial_{R}^{\{\alpha, \boldsymbol{\rho}\}} \theta^{\prime} \sim-\frac{\pi}{2} \delta\left(R-R_{0}\right) .
$$

The discontinuous character also explains the pole in the rotational coupling, since for an infinitesimal $\alpha \neq 0$ and $R$ $=R_{0}$ from Eqs. (19) and (9) we have $\psi_{2}=\left(\varphi_{2}-\varphi_{1}\right) / \sqrt{2}$. Hence the derivative of $\psi_{2}$ with respect to $\alpha$ tends to $-\infty$ on the left of $R_{0}$ and to $+\infty$ on the right. For a fixed $R$ value and small $\alpha$, and from Eqs. (15) and (18), we obtain

$$
\partial_{\rho_{\alpha}}^{\{\overline{\boldsymbol{R}}, \rho\}} \theta^{\prime}=-\partial_{\alpha}^{\{R, \boldsymbol{\rho}\}} \theta^{\prime} \sim \frac{-\delta_{0}}{2\left(R-R_{0}\right)} .
$$

Incidentally, we note that the $\delta$-function term in Eq. (12), which we found to be completely swamped by the pole in our calculations [10,19], has been eliminated in Eq. (21).

Second, both short and long range effects of the CI in the rotational coupling can be understood by introducing the angle $\beta$ between the $\boldsymbol{R}-\boldsymbol{R}_{0}$ and $\boldsymbol{\rho}$ vectors, and taking $R_{0}$ sufficiently large that we have, to a good approximation,

$$
\tan \beta=\frac{R|\alpha|}{R-R_{0}},
$$

which we can compare to

$$
\theta^{\prime}=\frac{1}{2} \tan ^{-1} \frac{\delta_{0}|\alpha|}{R_{0}-R}
$$

which is equivalent to Eq. (19) using the double $(0, \pi / 2)$ $-(\pi / 2, \pi)$ branch form of the arctangent function. The comparison yields

$$
\theta^{\prime}=\frac{1}{2} \tan ^{-1}\left(-\frac{\delta_{0}}{R} \tan \beta\right)
$$

We see that the transformation angle $\theta^{\prime}$ is a smooth function of $\beta$, whose derivative is

$$
\partial_{\beta}^{\{R, \boldsymbol{\rho}\}} \theta^{\prime}=-\frac{\delta_{0} R / 2}{\delta_{0}^{2} \sin ^{2} \beta+R^{2} \cos ^{2} \beta}
$$

and the singular term in Eq. (18) is due to

$$
\partial_{\alpha}^{\{R, \boldsymbol{\rho}\}} \theta^{\prime}=\frac{d \beta}{d \alpha} \partial_{\beta}^{\{R, \boldsymbol{\rho}\}} \theta^{\prime}=\operatorname{sgn}(\alpha) \frac{R\left(R-R_{0}\right)}{R^{2} \alpha^{2}+\left(R-R_{0}\right)^{2}} \partial_{\beta}^{\{R, \boldsymbol{\rho}\}} \theta^{\prime} .
$$

For $\alpha \rightarrow 0$ the pole at $R=R_{0}$ arises because of the corresponding pole in $d \beta / d \alpha$, so that to an infinitesimal increase in $\alpha$ from the zero value corresponds an increasingly larger increase in $\beta$ as $R \rightarrow R_{0}$. The long range rotational coupling arises because $\partial_{\beta}^{\{R, \boldsymbol{\rho}\}} \theta^{\prime} \rightarrow-\delta_{0} / 2 R$ as $\left|R-R_{0}\right| \rightarrow \infty$ (so that $\beta \rightarrow 0)$. For instance, in the $R \rightarrow+\infty$ limit Eq. (24) gives $\theta$ $\rightarrow \pi / 2-\delta_{0} \beta / 2 R$ and Eq. (9) reads

$$
\psi_{2}(\alpha) \approx-\varphi_{1}(0)+\frac{\delta_{0} \beta}{2 R} \varphi_{1}(0) \approx \frac{\delta_{0}|\alpha|}{2\left(R-R_{0}\right)} \psi_{1}(0)+\psi_{2}(0)
$$

so that the admixture of $\psi_{1}$ to $\psi_{2}$ decreases like $R^{-1}$ for fixed $\beta$ and like $\left(R-R_{0}\right)^{-1}$ for fixed $\alpha$.

The previous explanations can obviously be extended to the more general transformation (10).

\section{Elimination of the singular couplings}

We now deal with the practical aspect of devising a workable method to remove the singularities. From the description in the previous section, it follows that an appropriate procedure is to transform the basis using inversion of Eq. (9). This yields well-known expressions for the Hamiltonian matrix elements and couplings. For instance, in the laboratoryfixed frame, we have

$$
\begin{gathered}
H_{22}-H_{11}=(\cos 2 \theta)\left(E_{2}-E_{1}\right), \\
H_{12}=-(\sin 2 \theta)\left(E_{2}-E_{1}\right) / 2, \\
\left\langle\varphi_{1}\left|\partial_{R}^{\{r, \alpha, \boldsymbol{\rho}\}}\right| \varphi_{2}\right\rangle=\left\langle\psi_{1}\left|\partial_{R}^{\{\boldsymbol{r}, \alpha, \boldsymbol{\rho}\}}\right| \psi_{2}\right\rangle+\partial_{R}^{\{\alpha, \boldsymbol{\rho}\}} \theta, \\
\left\langle\varphi_{1}\left|\partial_{\alpha}^{\{r, R, \boldsymbol{\rho}\}}\right| \varphi_{2}\right\rangle=\left\langle\psi_{1}\left|\partial_{\alpha}^{\{\boldsymbol{r}, R, \boldsymbol{\rho}\}}\right| \psi_{2}\right\rangle+\partial_{\alpha}^{\{R, \boldsymbol{\rho}\}} \theta,
\end{gathered}
$$

and analogous expressions in the body-fixed frame. With suitable choices of the transformation angle $\theta$, this inverse transformation can then be employed to generate suitable functions $\varphi_{1}$ and $\varphi_{2}$ that vary smoothly, presenting nonsingular (nonadiabatic and Hamiltonian) couplings. In this respect, it is useful to summarize first what would be the main requirements of an appropriate procedure.

First, one should bear in mind that diabatic wave functions that exhibit no dynamical couplings at all cannot be obtained in general (see Refs. [26,7,12]). In fact this is not a hindrance: since in the semiclassical approach of Ref. [13] only systems of first order differential equations appear, there is no difficulty in dealing with both nonadiabatic and Hamiltonian couplings, provided they are regular. Hence, it is not advantageous to ask too much from the transformation.

Second, the singular behavior of the couplings poses a practical problem only when $\alpha$ is very small, so that we need to focus on only these grazing $\boldsymbol{R}$ trajectories. For other kinds of trajectory (at least when the angle-averaging approach of Ref. [13] is used), difficulties related to conical intersections can be avoided in most situations, because of the strong decrease of the first term in Eqs. (11) and (12) when $\alpha$ is large. This is why the transformation (28) is needed only for a narrow bundle of nuclear trajectories, depending upon the CI considered: in the example of Ref. [10], a reasonable domain 
is obtained for $\alpha<1^{0}$, whereas in the case treated in [19] it can be taken to be $\alpha<10^{0}$; usually, we find that the smaller $R_{0}$ is the larger the domain is.

Finally, unless the position of the $\mathrm{CI}$ is known to infinite precision, subtraction in Eq. (28) of a $\delta$-type peak $-\partial_{R}^{\{\alpha, \boldsymbol{\rho}\}} \theta$ from another $\delta$-type peak in $\left\langle\varphi_{1}\left|\partial_{R}^{\{r, \alpha, \boldsymbol{\rho}\}}\right| \varphi_{2}\right\rangle$ will yield a (generally asymmetric, and very narrow) double peak. This is of no practical importance, since the area under the double peak is zero, so that it can be safely ignored. In fact, it should be ignored, since it is so narrow that obtaining some (i.e., too few) points and interpolating through them is likely to yield spurious transitions. Similar considerations apply to the rotational coupling. The practical method would then simply ignore the region extremely close to the CI, use Eqs. (28) outside this region, and smoothly interpolate the resulting data.

In the following section we report our findings for the choices that seem most obvious from the preceding discussion. These recipes employ the following transformation angles in Eqs. (9) and (28).

(I) The analytical expression of Eq. (10) for the transformation angle $\theta$. This method was employed in our previous work (e.g., Refs. [10,19]).

(II) The transformation angle

$$
\theta^{\prime \prime}=-\int_{0}\left\langle\psi_{1}\left|\partial_{\rho_{\alpha}}^{\{\bar{r}, \overline{\boldsymbol{R}}, \rho\}}\right| \psi_{2}\right\rangle d \rho_{\alpha}
$$

using body-fixed expressions analogous to Eqs. (28).

(III) The transformation angle

$$
\theta^{\prime \prime \prime}=-\int_{0}\left\langle\psi_{1}\left|\partial_{R}^{\{r, \alpha, \boldsymbol{\rho}\}}\right| \psi_{2}\right\rangle d R
$$

Equations (29) and (30) provide straightforward extensions of the procedures usually employed in ion-atom collisions.

(IV) The analytical expression of Eq. (19) for the transformation angle $\theta^{\prime}$, where the parameters are obtained for a value of $\alpha$ sufficiently small.

\section{RESULTS AND DISCUSSION}

We now discuss, and illustrate, our findings in the implementation of techniques I-IV of Sec. II C.

We considered in Ref. [19] electronic transitions taking place in collisions between $\mathrm{N}^{5+}$ ions and $\mathrm{H}_{2}$ molecules in the energy range $50 \mathrm{eV} \mathrm{amu}^{-1}-6 \mathrm{keV} \mathrm{amu}^{-1}$. For these impact energies, we can assume that the $\mathrm{N}^{5+}\left(1 s^{2}\right)$ core does not change during the transitions, and the active electron is represented by (self-consistent-field-type) molecular orbitals. Orbital energies and nonadiabatic couplings were obtained $a b$ initio with the MELDR program [23-25]. We do not show the complete energy diagram, because it is not relevant here, and it is rather complicated [19], displaying pseudocrossings and crossings of the surfaces. As a consequence of these complications, and as mentioned in the Introduction, it is very useful to study first the case of a linear nuclear configuration in order to select the signs of the nonadiabatic couplings - since, as is well known, ab initio programs such

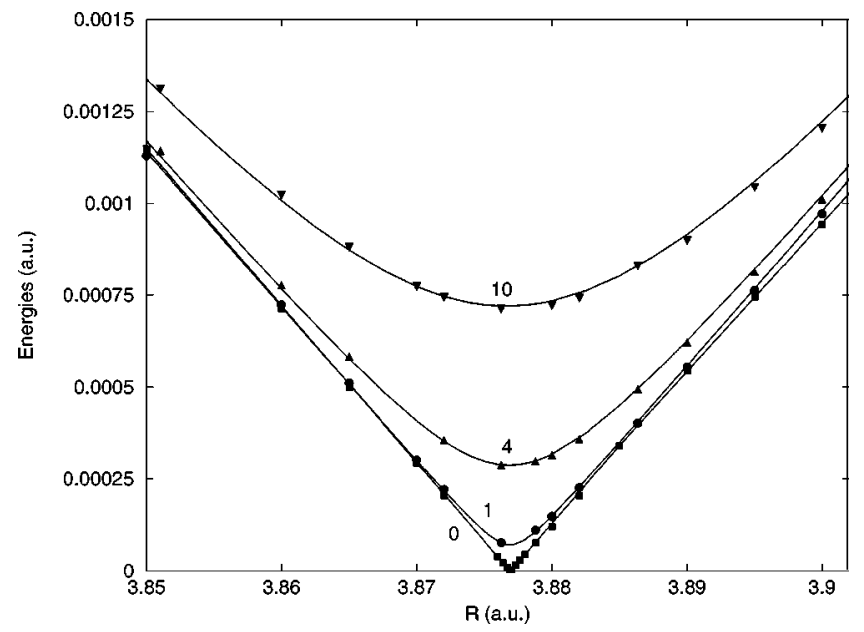

FIG. 1. Analytical bilinear model (I) [Eq. (13)] (lines) and $a b$ initio calculated (symbols) adiabatic energies in the vicinity of the $\mathrm{CI}$ between the states of the $\mathrm{NH}_{2}{ }^{5+}$ quasimolecule dissociating into $\mathrm{N}^{4+}\left(1 s^{2} 2 p_{z}\right)+\mathrm{H}_{2}{ }^{+}\left(\sigma_{g}\right)$ and $\mathrm{N}^{4+}\left(1 s^{2} 2 p_{x}\right)+\mathrm{H}_{2}{ }^{+}\left(\sigma_{g}\right)$, as functions of the projectile distance $R$, obtained for four values of the relative angle $\alpha=0^{\circ}, 1^{\circ}, 4^{\circ}$, and $10^{\circ}$. Symbols: squares $\left(0^{\circ}\right)$, circles $\left(1^{\circ}\right)$, up triangles $\left(4^{\circ}\right)$, down triangles $\left(10^{\circ}\right)$.

as MELDR provide only the absolute values of these matrix elements. As expected, one finds numerical difficulties in the direct integration of the dynamical equations in the vicinity of CIs, and the transition probabilities obtained in the adiabatic basis behave erratically. Hence, we shall focus here on the characteristics of the quasisingular and transformed molecular data near an isolated conical point, although we keep in mind more complicated situations.

The two selected energy surfaces correspond to two molecular states dissociating into $\mathrm{N}^{4+}\left(3 p_{z}\right)+\mathrm{H}_{2}{ }^{+}$and $\mathrm{N}^{4+}\left(3 p_{x}\right)+\mathrm{H}_{2}{ }^{+}$, which lead to sharing between the electron capture channels as projectile and target separate. The surfaces $E_{1}$ and $E_{2}$ exhibit a CI, for $R_{0} \approx 4$ a.u. and $\alpha=0$, which is considerably wider than the one considered in Ref. [10], and therefore better adapted as a benchmark to compare different techniques.

For linear $\boldsymbol{R}$ trajectories with $\alpha=0^{\circ}, 1^{\circ}, 4^{\circ}, 10^{\circ}$, we display in Fig. 1 some calculated values of the adiabatic energy differences $E_{2}-E_{1}$ near the CI. For $\alpha=1^{\circ}, 4^{\circ}, 10^{\circ}$, the corresponding radial couplings $\left\langle\psi_{1}\left|\partial_{R}^{\{\boldsymbol{r}, \alpha, \boldsymbol{\rho}\}}\right| \psi_{2}\right\rangle$ and rotational couplings $\left\langle\psi_{1}\left|R^{-1} \partial_{\alpha}^{\left\{\boldsymbol{r}, R, \boldsymbol{\rho}_{\}}\right.}\right| \psi_{2}\right\rangle$ are presented in Figs. 2 and 3, respectively. From the size and shape of the couplings, which is qualitatively the same as in Ref. [10], it is obvious that a change of basis is required in order to treat the dynamics. For this purpose, we considered the recipes I-IV of the previous section, which make use of the inverse of the transformation (9) and application of Eqs. (28).

Method I. This procedure was employed in Refs. [10,19], and uses a fit of the data for the energy difference and the dynamical couplings, near the $\mathrm{CI}$, to the analytical expressions of Eqs. (13), (11), and (12), respectively. In practice, this nonlinear fit is a delicate numerical step, because of the number of parameters involved, and the fact that it requires an iterative procedure, with a preliminary gross fit of the energy differences, followed by a fine tuning using the non- 


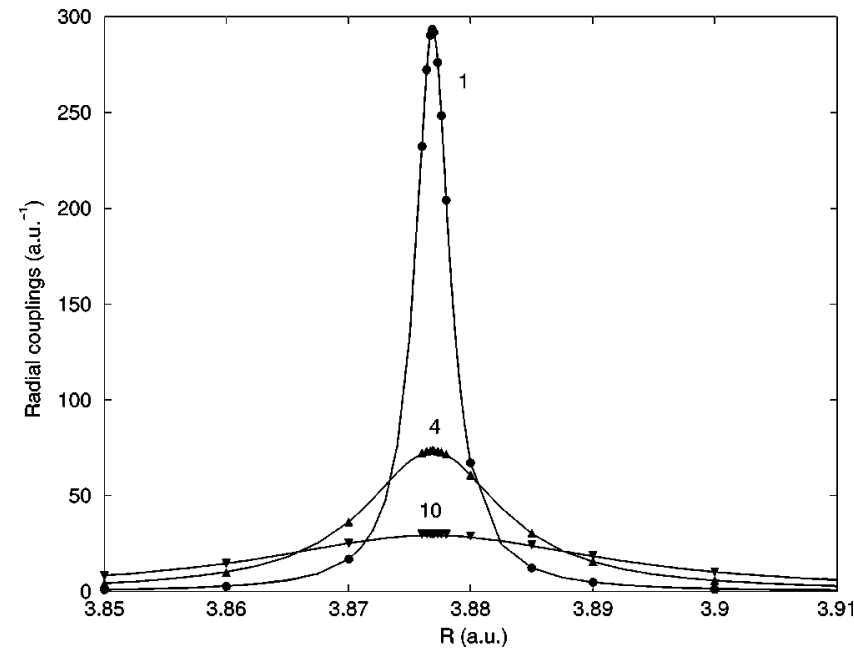

FIG. 2. Comparison between analytical $\partial_{R}^{\{\alpha, \boldsymbol{\rho}\}} \theta$, and ab initio calculated $\left\langle\psi_{1}\left|\partial_{R}^{\{\boldsymbol{r}, \alpha, \boldsymbol{\rho}\}}\right| \psi_{2}\right\rangle$ radial couplings of Eq. (11), as functions of $R$, for three values of the relative angle $\alpha=1^{\circ}, 4^{\circ}$, and $10^{\circ}$, and with the labels as in Fig. 1.

adiabatic couplings, reconsideration of the energies, and so on. As a consequence, application to the case of partially overlapping CIs is nearly hopeless.

In the present case, the fitted parameters are $R\{0\}$ $=3.8769242, \quad a=0.04228, \quad b=1.286079 \times 10^{-5}, \quad$ and $c=5.325962 \times 10^{-4}$ a.u. To show the reliability of the fits near the CI, we have included in Figs. 1-3 the values obtained from the analytical expressions of the first terms of the RHS of Eqs. (13), (11), and (12). In the narrow $\boldsymbol{R}$ domain about the CI considered in Figs. 1 and 2, exact and analytical (parametrized) curves for the energy differences and radial couplings are very close. On the other hand, and in apparent sharp contrast with our previous findings in Ref. [10], there remain in Fig. 3 sizable discrepancies between the $a b$ initio rotational coupling and the parametrized forms. It should be stressed that this is not a liability of the method, and in fact

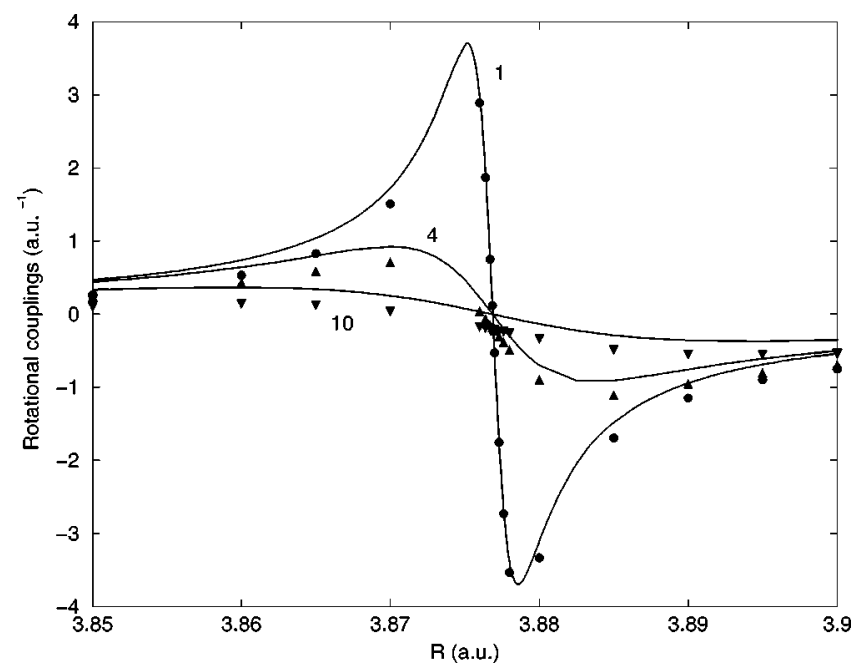

FIG. 3. Analytical $R^{-1} \partial_{\alpha}^{\{R, \boldsymbol{\rho}\}} \theta$ and calculated $\left\langle\psi_{1}\left|R^{-1} \partial_{\alpha}^{\{\boldsymbol{r}, R, \boldsymbol{\rho}\}}\right| \psi_{2}\right\rangle$ rotational couplings of Eq. (12), as functions of $R$. Same symbols and angles as in Fig. 2.

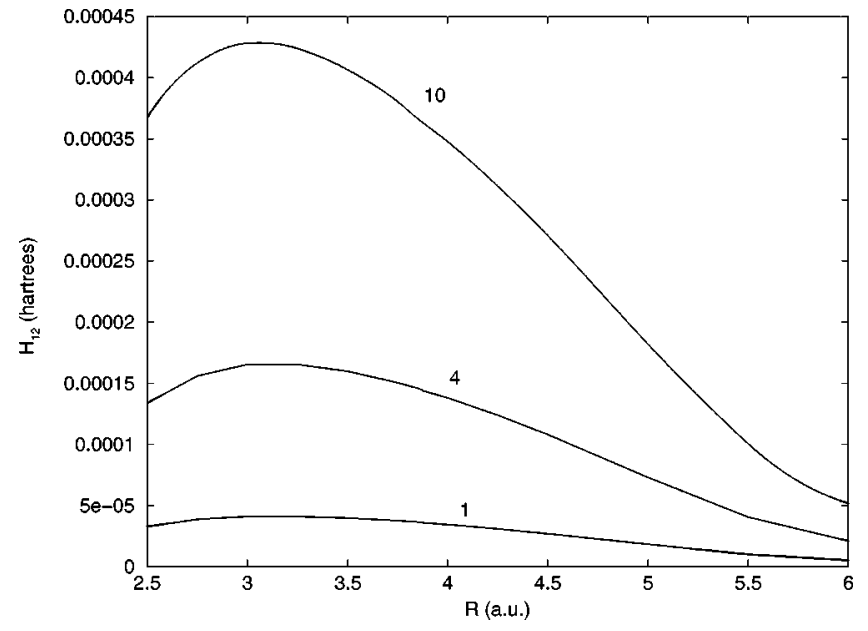

FIG. 4. $H_{12}$ Hamiltonian coupling between the states $\left\{\varphi_{1,2}\right\}$ obtained from Eq. (28), as functions of $R$, for the same three angles $\alpha$ as in previous figures.

it is a rather common feature that can be expected from Eq. (12), because of the existence of non-negligible "regular terms" near the CI; this point will be further discussed below. Furthermore, non-negligible regular terms in the radial coupling are also expected for other molecular states exhibiting asymptotic Stark delocalization (in the so-called Nikitin-Demkov model) or residual interactions [22].

As in Ref. [10], the transformed molecular data obtained from Eqs. (28) behave regularly as functions of $\alpha$ and $R$. In the CI region, the energy differences $H_{22}-H_{11}$ are extremely close to $E_{2}-E_{1}$ given in Fig. 1 for $\alpha=0^{\circ}$ and $R<R_{0}$ and to $E_{1}-E_{2}$ for $\alpha=0^{\circ}$ and $R>R_{0}$, as expected. We show in Fig. 4 the Hamiltonian couplings $H_{12}$ which are tiny in the whole $\boldsymbol{R}$ domain. The nonadiabatic couplings are smooth, except that, as explained in the previous section, they exhibit ineffectual peaks at the CI. Thus, the radial coupling $\left\langle\varphi_{1}\left|\partial_{R}^{\{\boldsymbol{r}, \alpha, \boldsymbol{\rho}\}}\right| \varphi_{2}\right\rangle$ exhibits an extremely narrow double peak structure, which should be ignored in actual dynamical calculations. When those peaks are eliminated by the expeditious way of interpolating through them, the ensuing radial couplings are shown in Fig. 5, and the rotational couplings $\left\langle\varphi_{1}\left|R^{-1} \partial_{\alpha}^{\{\boldsymbol{r}, R, \boldsymbol{\rho}\}}\right| \varphi_{2}\right\rangle$ in Fig. 6. Next, to explain the smooth but sizable rotational term in the latter figure, we have displayed in Fig. 7, for an $\boldsymbol{R}$ trajectory with $\alpha=10^{\circ}$, the three rotational-type matrix elements $\left\langle\psi_{1}\left|R^{-1} \partial_{\alpha}^{\{\boldsymbol{r}, R, \boldsymbol{\rho}\}}\right| \psi_{2}\right\rangle$, $\left\langle\psi_{1}\left|R^{-1} \partial_{\rho_{\alpha}}^{\{\overline{\boldsymbol{r}}, \overline{\boldsymbol{R}}, \rho\}}\right| \psi_{2}\right\rangle$, and $\left\langle\psi_{1}\left|R^{-1} i L_{y}^{-}\right| \psi_{2}\right\rangle$, fulfilling Eq. (15), together with the parametrized term $R^{-1} \partial_{\alpha}^{\{R, \boldsymbol{\rho}\}} \theta$ [see Eq. (11)] and the coupling $\left\langle\varphi_{1}\left|R^{-1} \partial_{\alpha}^{\{\boldsymbol{r}, R, \boldsymbol{\rho}\}}\right| \varphi_{2}\right\rangle$. Figure 7 shows that we have

$$
\left\langle\psi_{1}\left|\partial_{\alpha}^{\{\boldsymbol{r}, R, \boldsymbol{\rho}\}}\right| \psi_{2}\right\rangle=\left\langle\psi_{1}\left|\partial_{\rho_{\alpha}}^{\{\overline{\boldsymbol{r}}, \overline{\boldsymbol{R}}, \rho\}}\right| \psi_{2}\right\rangle+\left\langle\psi_{1}\left|i L_{y}^{-}\right| \psi_{2}\right\rangle
$$

according to Eq. (15), and the approximate relations for $R>3$ a.u. 


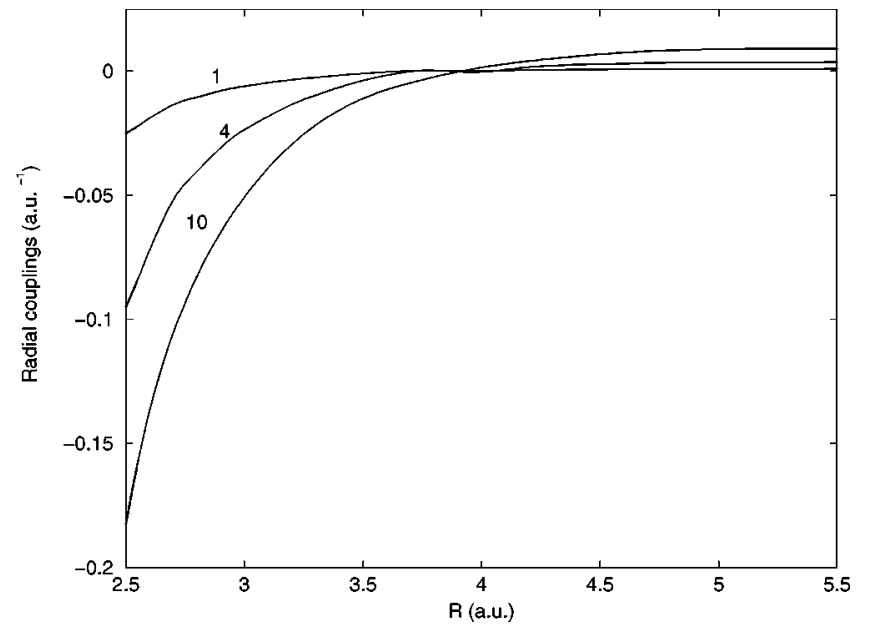

FIG. 5. Radial couplings $\left\langle\varphi_{1}\left|\partial_{R}^{\{\boldsymbol{r}, \alpha, \boldsymbol{\rho}\}}\right| \varphi_{2}\right\rangle$ of Eq. (28), as functions of $R$, for $\alpha=1^{\circ}, 4^{\circ}$, and $10^{\circ}$.

$$
\begin{gathered}
\partial_{\alpha}^{\{R, \boldsymbol{\rho}\}} \theta \approx\left\langle\psi_{1}\left|\partial_{\rho_{\alpha}}^{\left\{\bar{r}, \overline{\boldsymbol{R}}, \rho_{\}}\right\}}\right| \psi_{2}\right\rangle, \\
\left\langle\varphi_{1}\left|\partial_{\alpha}^{\{\boldsymbol{r}, R, \boldsymbol{\rho}\}}\right| \varphi_{2}\right\rangle \approx\left\langle\psi_{1}\left|i L_{y}^{-}\right| \psi_{2}\right\rangle .
\end{gathered}
$$

The smooth behavior of the last two terms in Eq. (32) stands in contrast to the quasisingular behavior of the others, discussed in Sec. II B. From Eqs. (32), we see that, for the present example, the "regular terms" of Eq. (16) are very small near the CI, and those of Eq. (12) are close to $\left\langle\psi_{1}\left|i L_{y}^{-}\right| \psi_{2}\right\rangle$. However, this is not the case for $R<3$ a.u. nor for wider angles. We have also found that it is not the case either for other systems where CIs appear at short $R_{0}$, such as $\mathrm{K}^{+}+\mathrm{H}_{2}$ collisions, where $\left\langle\psi_{1}\left|\partial_{\rho_{\alpha}}^{\{\overline{\boldsymbol{r}}, \overline{\boldsymbol{R}}, \rho\}}\right| \psi_{2}\right\rangle$ contains sizable "regular terms"' in Eq. (16).

Method II. The second possibility that we have tested makes use of Eq. (29) to define the transformation angle to be used in the body-fixed version of Eqs. (28). For fixed $\boldsymbol{\rho}$ and a grid of $\boldsymbol{R}$ values, it employs numerical integration of the exact nonadiabatic rotational coupling $\left\langle\psi_{1}\left|\partial_{\rho_{\alpha}}^{\{\bar{r}, \overline{\boldsymbol{R}}, \rho\}}\right| \psi_{2}\right\rangle$ as

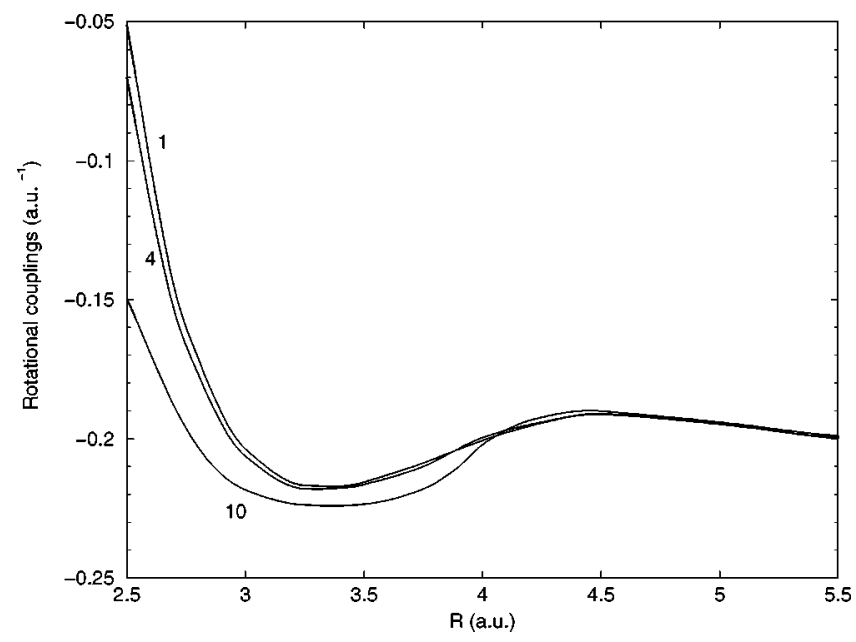

FIG. 6. Rotational couplings $\left\langle\varphi_{1}\left|R^{-1} \partial_{\alpha}^{\{\boldsymbol{r}, R, \boldsymbol{\rho}\}}\right| \varphi_{2}\right\rangle$ of Eq. (28), as functions of $R$, for $\alpha=1^{\circ}, 4^{\circ}$, and $10^{\circ}$.

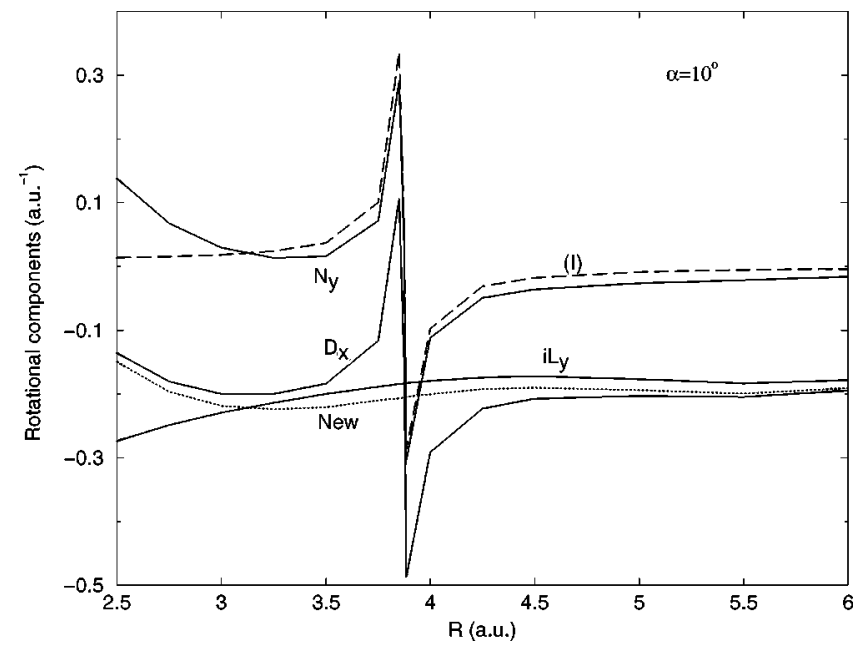

FIG. 7. Total $\left(D_{x}\right)\left\langle\psi_{1}\left|R^{-1} \partial_{\alpha}^{\{\boldsymbol{r}, R, \boldsymbol{\rho}\}}\right| \psi_{2}\right\rangle$, singular component $\left(N_{y}\right)\left\langle\psi_{1}\left|R^{-1} \partial_{\rho_{\alpha}}^{\{\overline{\boldsymbol{r}}, \overline{\boldsymbol{R}}, \rho\}}\right| \psi_{2}\right\rangle$, regular component $\left(i L_{y}\right)$, [Eqs. (7) and (15)], analytical (I) $R^{-1} \partial_{\alpha}^{\{R, \boldsymbol{\rho}\}} \theta$ [Eq. (11)], and the (new) $\left\langle\varphi_{1}\left|R^{-1} \partial_{\alpha}^{\{\boldsymbol{r}, R, \boldsymbol{\rho}\}}\right| \varphi_{2}\right\rangle$ rotational couplings obtained for an angle $\alpha=10^{\circ}$ as functions of $R$.

a function of $\rho_{\alpha}$. As would be expected from Eqs. (29) and (32), we obtain that the angles $\theta$ and $\theta^{\prime \prime}$ obtained in methods I and II are essentially the same for $R>3$ a.u.

The direct integration procedure employed in this second method has the advantage of being parameter-free, so that no difficult fitting procedure is required as in method I. However, we have found that it is very hard to obtain accurate values for the radial couplings, even outside the CI region. The reason is that it requires the evaluation of a large number of values of the $\left\langle\psi_{1}\left|\partial_{\rho_{\alpha}}^{\overline{\boldsymbol{r}}, \overline{\boldsymbol{R}}, \rho\}}\right| \psi_{2}\right\rangle$ interactions to be integrated with respect to $\alpha$ and then differentiated with respect to $R$. This liability renders the method useful only when there is some reason to believe that these couplings are negligible. Moreover, the method also has the inconvenience that the Hamiltonian couplings (28) are large for $\boldsymbol{R}$ values that are far from $\boldsymbol{R}_{0}$. This is not desirable in the treatment of the dynamics, since it would require a passage from $\varphi_{1}, \varphi_{2}$ to the original $\psi_{1}, \psi_{2}$ basis, and this is awkward unless $\lim _{R \rightarrow \infty} \theta(R)$ $=\pi / 2$ to a good enough approximation.

Method III. Similar difficulties are met when employing the third procedure of Eq. (30), in which the radial coupling is integrated instead of the rotational one. This procedure is very close to the previous one, but is not completely identical, since, as is easily shown by using the argument of $[26,7,12]$ in the body-fixed frame, an orthogonal transformation (9) can get rid of only the irrotational part of the coupling $\left\langle\psi_{1}\left|\nabla_{\bar{R}}\right| \psi_{2}\right\rangle$. Nevertheless, for the present example the two methods are essentially equivalent near the CI. We thus conclude that the methods of integration of the exact couplings between the adiabatic wave functions are not well adapted to treating ion-molecule collisions near CIs, unless one can neglect the residual couplings.

Method $I V$. The fourth possibility that we have applied, and that we propose here as a reasonably satisfactory solution to the problem, is to use the simplified limit expression of Eq. (19) to define the transformation angle $\theta$. The param- 
eters $R_{\alpha}, \delta_{\alpha}$ appearing in this equation can be obtained, e.g., by a calculation of the radial coupling $\left\langle\psi_{1}\left|\partial_{R}^{\{r, \alpha, \boldsymbol{\rho}\}}\right| \psi_{2}\right\rangle$ for a selected trajectory with $\alpha$ sufficiently small. From Eq. (17), we can take the position of the peak of the coupling to yield $R_{\alpha}$ and its height $\left(2 \delta_{\alpha} \alpha\right)^{-1}$. In all cases considered, this method is numerically stable, because it depends only upon two rather unconnected parameters, so that extension to the case of nonisolated CIs does not present the unsurmountable difficulties of our previous approach.

In the present case, it is enough to take $\alpha=1^{\circ}$, which gives $R_{1}\left(=R_{\alpha=1^{\circ}}\right)=3.87685$ and $\delta_{1}=0.0975$ in atomic units. These values agree reasonably with the estimates $R_{0}$ $-R_{1} \approx b R_{0} \alpha / a$ and $\delta_{1} \approx \delta_{0}=2 c R_{0} / a$, with the values of $R_{0}, a, b, c$ obtained for method I.

As may be expected, since the parameters in Eqs. (17) and (18) are obtained for $\alpha=1^{\circ}$, the fits of the molecular data are better for this particular trajectory than those obtained with the more general expressions (11) and (12) with the parameters fitted at the CI. Nevertheless, the changes are unnoticeable in Figs. 1-6, because they only affect unimportant features of the couplings, such as the double peaked structures of the radial couplings, which are eliminated in actual calculations. Hence, the results of our figures can be taken to be identical, to all practical purposes, for methods I and IV, at least for $\alpha \leqslant 10^{\circ}$.

Finally, as stressed in the Introduction, an undesirable feature of the singular rotational couplings is that they extend to regions far from the CI. Actually, it is common that they extend to $R$ values such that the bilinear expressions used to derive Eqs. (12), (16), and (18) in Ref. [10] are no longer good enough approximations. In such cases, it is impossible to separate "normal" rotational couplings from the tail of those due to the CI. The solution to this problem is straightforward when, as is the case for Eqs. (10) and (19), the transformation angle $\theta$ goes from 0 to $\pi / 2$ in the near neighborhood of the CI, so that a little away from this region, a passage from the $\varphi_{1}, \varphi_{2}$ basis to the more appropriate adiabatic functions $\psi_{1}, \psi_{2}$ obtains. In our opinion, this is an asset of method IV.

\section{CONCLUSIONS}

The results of the present work are twofold. First, we have presented a detailed analysis of the characteristics of nonadiabatic couplings near CIs that appear in the application of the SEIKON method of Ref. [13] to ion-diatomic molecule processes. This applies especially to the expressions for the rotational terms [Eqs. (7), (16), (18), and (22)-(27)], which were not given in our previous work [10]. In particular, we have shown the physical origin of the couplings due to CIs appearing for collinear geometries of the nuclear frame. As could be expected, since the relevant adiabatic wave functions abruptly exchange their character at a conical point, singularities appear when differentiating this discontinuous behavior. Thus, a $\delta$ function appears for the radial coupling, which follows as the limit of typical Lorentzian peaks at pseudoscrossings in the limit of zero energy gap [22]. Another awkward singularity is due to the change of sign that accompanies one of the two wave functions as their character is interchanged. This gives rise to a pole in the rotational coupling, which extends to relatively large distances from the CI, because the admixture of adiabatic wavefunctions when the nuclear configuration is bent by an angle $\alpha$ is proportional to this angle.

Second, we found that our previous technique (called method I) to remove the singularities, using the transformations (28) with the angle given by Eq. (10), is numerically complicated and unstable with respect to errors in the parameters. This is because Eq. (10) involves four parameters that are strongly coupled variables in the fitting procedure. Consequently, method I is difficult to apply to situations where CIs are not clearly isolated, and even then it is prone to numerical errors. Because of this, three alternative methods (called methods II-IV) have been considered here.

Two of these techniques (II and III) consist in generalizing the procedure employed for the analogous case of ionatom collisions, where one integrates the culprit dynamical couplings, rather than an analytical expression. This is not a diabatization procedure, which, as is well known, is not possible for triatomic systems - and not even desirable, as discussed in Sec. II C. Nevertheless, although integration of either radial [Eq. (30)] or rotational [Eq. (29)] couplings near CIs removes the singularities in the couplings, the procedures were not found to be completely satisfactory because of the difficulty in obtaining the residual nonadiabatic interactions. However, when these interactions can be neglected, methods II and III are satisfactory, especially as there is wide experience in their application to the ion-atom case.

We then considered an alternative recipe (method IV) that allows accurate calculation of residual couplings. The method is a simplified version of I that avoids complicated fittings, by restricting our attention to the bundle of trajectories for which the singularities are really a problem. For these trajectories, one uses the transformations of Eqs. (28) with the angle (19), which contains only two parameters that are very easily obtained (see Sec. III). The procedure is stable and can be applied to more complicated issues. In our opinion, it provides a satisfactory solution to the practical problem, as it can be applied to all cases we have treated, although more calculations are obviously necessary to confirm this in full generality.

\section{ACKNOWLEDGMENT}

This work was partially supported by DGICYT Project No. PB96-0056.
[1] L. F. Errea, A. Macías, L. Méndez, and A. Riera, J. Phys. B 32, 4065 (1999).

[2] L. F. Errea, A. Macías, L. Méndez, I. Rabadán, and A. Riera, J. Phys. B 33, L615 (2000).
[3] D. Elizaga, L. F. Errea, A. Macías, L. Méndez, A. Riera, and A. Rojas, J. Phys. B 33, 2037 (2000).

[4] D. E. Dombrowski, E. B. Desknis and M. A. Pick, Atomic and Plasma-Material Interaction Data for Fusion (International 
Atomic Energy Agency, Vienna, 1994), Vol. 5, p. 19.

[5] H. C. Longuet-Higgins, U. Opik, M. H. L. Pryce, and R. A. Sack, Proc. R. Soc. London, Ser. A 244, 1 (1958).

[6] M. Baer, S. H. Lin, A. Alijah, S. Adhikari, and D. Billing, Phys. Rev. A 62, 032506 (2000).

[7] C. A. Mead and D. G. Truhlar, J. Chem. Phys. 70, 2284 (1979).

[8] M. V. Berry, Proc. R. Soc. London, Ser. A 392, 45 (1984).

[9] X. Wu, R. E. Wyatt, and M. D'Mello, J. Chem. Phys. 101, 2953 (1994).

[10] D. Elizaga, L. F. Errea, A. Macías, L. Méndez, A. Riera, and A. Rojas, J. Phys. B 32, L697 (1999).

[11] C. A. Mead, J. Chem. Phys. 78, 807 (1983).

[12] N. Baer and C. Y. Ng, Adv. Chem. Phys. 82, 1 (1992).

[13] L. F. Errea, J. D. Gorfinkiel, A. Macías, and A. Riera, J. Phys. B 30, 3855 (1997).

[14] D. Elizaga, L. F. Errea, J. D. Gorfinkiel, C. Illescas, L. Méndez, A. Macías, A. Riera, and A. Rojas, Phys. Scr. T 80, 187 (1999).

[15] D. Elizaga, L. F. Errea, J. D. Gorfinkiel, C. Illescas, L. Méndez, A. Macías, A. Riera, A. Rojas, O. J. Kroneisen, T. Kirchner, H. J. Lüdde, A. Henner, and R. M. Driezler, J. Phys. B 32,
857 (1999).

[16] L. F. Errea, J. D. Gorfinkiel, A. Macías, L. Méndez, and A. Riera, J. Phys. B 32, 1705 (1999).

[17] L. F. Errea, J. D. Gorfinkeil, C. Harel, H. Jouin, A. Macías, L. Méndez, B. Pons, and A. Riera, J. Phys. B 33, 3107 (2000).

[18] B. H. Bransden and M. H. C. McDowell, Charge Exchange and the Theory of Ion-Atom Collisions (Oxford Science Publications, Oxford, 1992).

[19] L. F. Errea, A. Macías, L. Méndez, I. Rabadán, A. Riera, and P. Sanz, Int. J. Quantum Chem. (to be published).

[20] G. Lubinski, Z. Juhasz, R. Morganstern, and R. Hoekstra, J. Phys. B 33, 5275 (2000).

[21] F. T. Smith, Phys. Rev. 179, 111 (1969).

[22] A. Riera, J. Mol. Struct.: THEOCHEM 300, 93 (1993).

[23] E. R. Davidson, in Modern Techniques in Computational Chemistry, edited by E. Clementi (ESCOM, Leiden, 1990).

[24] J. F. Castillo, L. F. Errea, A. Macías, L. Méndez, and A. Riera, J. Chem. Phys. 103, 2113 (1995).

[25] L. F. Errea, J. D. Gorfinkeil, E. S. Kryachko, A. Macías, L. Méndez, and A. Riera, J. Chem. Phys. 106, 172 (1997).

[26] M. Baer, Chem. Phys. Lett. 35, 112 (1975). 